\title{
Релаксация возбужденных состояний мышьяка в деформированном германии
}

\author{
(C) К.А. Ковалевский ${ }^{1}$, Ю.Ю. Чопорова ${ }^{2,3}$, Р.Х. Жукавин ${ }^{1}$, Н.В. Абросимов ${ }^{4}$, С.Г. Павлов ${ }^{5}$, \\ H.-W. Hübers ${ }^{5,6}$, В.В. Цыпленков ${ }^{1}$, В.Д. Кукотенко ${ }^{2,3}$, Б.А. Князев ${ }^{2,3}$, В.Н. Шастин ${ }^{1}$ \\ ${ }^{1}$ Институт фозики микроструктур Российской академии наук, \\ 603950 Нижний Новгород, Россия \\ ${ }^{2}$ Институт ядерной физики им. Г.И. Будкера Сибирского отделения Российской академии наук, \\ 630090 Новосибирск, Россия \\ ${ }^{3}$ Новосибирский национальный исследовательский государственный университет, \\ 630090 Новосибирск, Россия \\ ${ }^{4}$ Leibniz Institute of Crystal Growth, \\ 12489 Berlin, Germany \\ ${ }^{5}$ Institute of Optical Sensor Systems, German Aerospace Center (DLR), \\ 12489 Berlin, Germany \\ ${ }^{6}$ Institut für Physik, Humboldt-Universität zu Berlin, \\ 12489 Berlin, Germany \\ E-mail: atan4@yandex.ru \\ Поступила в Редакцию 15 апреля 2020 г. \\ В окончательной редакции 21 апреля 2020 г. \\ Принята к публикации 21 апреля 2020 г.
}

Исследованы времена релаксации нижних p-состояний донора мышьяка в деформированном вдоль кристаллографического направления [111] кристалле германия. Измерения выполнялись методом накачказондирование с использованием излучения лазера на свободных электронах. Накачка состояний осуществлялась из основного состояния $1 s\left(A_{1}\right)$. Измеренное время распада состояния $2 p_{0}$ составляет 1.3 нс, $3 p_{0}-$ не более $0.2 \mathrm{Hc}, 2 p_{ \pm}-0.4$ нс. Показано, что относительно высокий темп релаксации состояния $2 p_{ \pm}$ обусловлен взаимодействием с внутридолинными ТА фононами.

Ключевые слова: германий, мышьяк, одноосная деформация, накачка-зондирование, внутрицентровое оптическое возбуждение, фононы.

DOI: $10.21883 /$ FTP.2020.10.49959.41

\section{1. Введение}

В последнее время новая волна интереса к свойствам мелких примесей в полупроводниках [1], в частности к исследованию релаксации возбужденных состояний, связана с появлением новых задач квантовой радиофизики [2] и квантовых вычислений [3], а также новых экспериментальных возможностей для прямого измерения ультрабыстрой и в то же время спектрально разрешенной динамики неравновесных носителей заряда [4].

Первые экспериментальные результаты по временам релаксации доноров и акцепторов в недеформированном германии были получены еще в 70-е годы с использованием комнатного теплового излучения в качестве возбуждающего источника, в то время как зондирующим было излучение лампы обратной волны [5]. Позднее с применением оптического затвора для уменьшения длительности импульса излучения лазера на свободных электронах (ЛСЭ) до нс было получено время фотоотклика $p$-Ge с концентрацией акцепторов $N_{a}=10^{15} \mathrm{~cm}^{-3}$ и компенсацией $50 \% \sim 1.7$ нс [6]. Появление многопользовательских установок типа „накачка-зондирование“ при инфракрасных ЛСЭ, имеющих длительности импульса излучения в диапазоне $10-100$ пс, позволило зна- чительно расширить экспериментальные возможности. В частности, было исследовано время захвата в $p$-Ge c различными уровнями легирования и компенсации [7], что позволило на основе полученных данных определить параметры, необходимые для уменьшения времени отклика германиевого детектора, и достичь рекордно коротких откликов фотопроводимости (150 пс) [4].

Методом накачка-зондирование в $n$-Ge были получены времена релаксации самых нижних возбужденных состояний (150-250 пс) в донорах сурьмы и мышьяка $[8,9]$, а также время релаксации (> 1нс) при различных интенсивностях накачки в зону проводимости [10]. Одноосная деформация вдоль направления [111] приводит к понижению симметрии, и при давлении > 300 бар нижние состояния доноров связаны с долинами только одного из направлений [111]. Такая модификация примесных состояний исключает участие междолинных фононов в процессах внутрицентровой релаксации, играющих важную роль в недеформированных кристаллах. В соответствии с этим следует ожидать существенных изменений времен релаксации. Данная работа имеет целью экспериментальное исследование времени релаксации возбужденных состояний доноров мышьяка в германии при криогенных температурах 
методом накачка-зондирование при наличии одноосной деформации кристалла вдоль кристаллографического направления [111].

\section{2. Эксперимент}

Кристаллы германия были выращены методом Чохральского с концентрацией мышьяка $N_{0}=10^{15} \mathrm{~cm}^{-3}$ и малой компенсацией $\left(\sim 10^{12} \mathrm{~cm}^{-3}\right)$. Образец имел размеры $2 \times 5 \times 7$ мм. Угол между полированными гранями $5 \times 7$ мм составлял $\sim 1.5^{\circ}$. Образец помещался в проточный гелиевый криостат Janis ST-100 с окнами TPX (полоса пропускания $\geq 15$ мкм) при температуре $T \approx 4 \mathrm{~K}$. Давление к образцу прикладывалось к грани $2 \times 5$ мм с помощью рычага и составляло $S \approx 2.5$ кбар. Bсе измерения проводились на экспериментальной установке накачки-зондирования при Сибирском центре синхротронного и терагерцового излучения [11]. В качестве источника терагерцового (ТГц) излучения использовался ЛСЭ NovoFEL. В данном эксперименте была задействована первая очередь ЛСЭ с возможностью перестройки в диапазоне $\lambda=90-220$ мкм, длительность импульса $\sim(100-150)$ пс, частота повторения в квазинепрерывном режиме 5.6 МГц. Выходное излучение ослаблялось с помощью поляризатора и делилось на пучок накачки и пучок зондирования с помощью делителя на основе лавсана в соотношении 20:1. Пучок зондирования проходил через линию задержки, а время прихода импульса зондирующего пучка после импульса накачки варьировалось в автоматическом режиме от отрицательных значений 1 нс до положительных значений задержки $\tau=4$ нс. Пучки фокусировались таким образом, что диаметр пятна на поверхности образца составлял $D=1$ мм для пучка зондирования и $D=2$ мм для пучка накачки. Угол между пучками $\alpha \approx 15^{\circ}$. После прохождения образца излучение собиралось линзой на приемнике Голея. При помощи системы из двух поляризаторов в каждом канале мощность пучка можно было изменять независимо с сохранением поляризации. Для уменьшения влияния излучения накачки на сигнал приемника в канале зондирования поляризации в пучках ставились под углом 90. Дополнительно в канале зондирования (перед приемником) стоял поляризатор в позиции, при которой прохождение излучения накачки минимально. Излучение накачки модулировалось с помощью прерывателя на частоте 15 Гц, а исследуемый сигнал подавался на синхронный усилитель (SR-830 lock-in amplifier). Частота излучения ЛСЭ в эксперименте соответствовала возбуждению $p$-состояний донора As в деформированном германии из основного состояния.

При фотовозбуждении самого нижнего возбужденного состояния $2 p_{0}$ (рис. 1) (энергия связи 4.75 мэВ, $1 s\left(\Gamma_{1}\right) \rightarrow 2 p_{0}$, при $S \approx 2.5$ кбар длина волны накачки $\lambda=190$ мкм) отклик (временна́я зависимость интенсивности прошедшего через образец пробного сигнала от задержки по отношению к накачивающему импульсу)

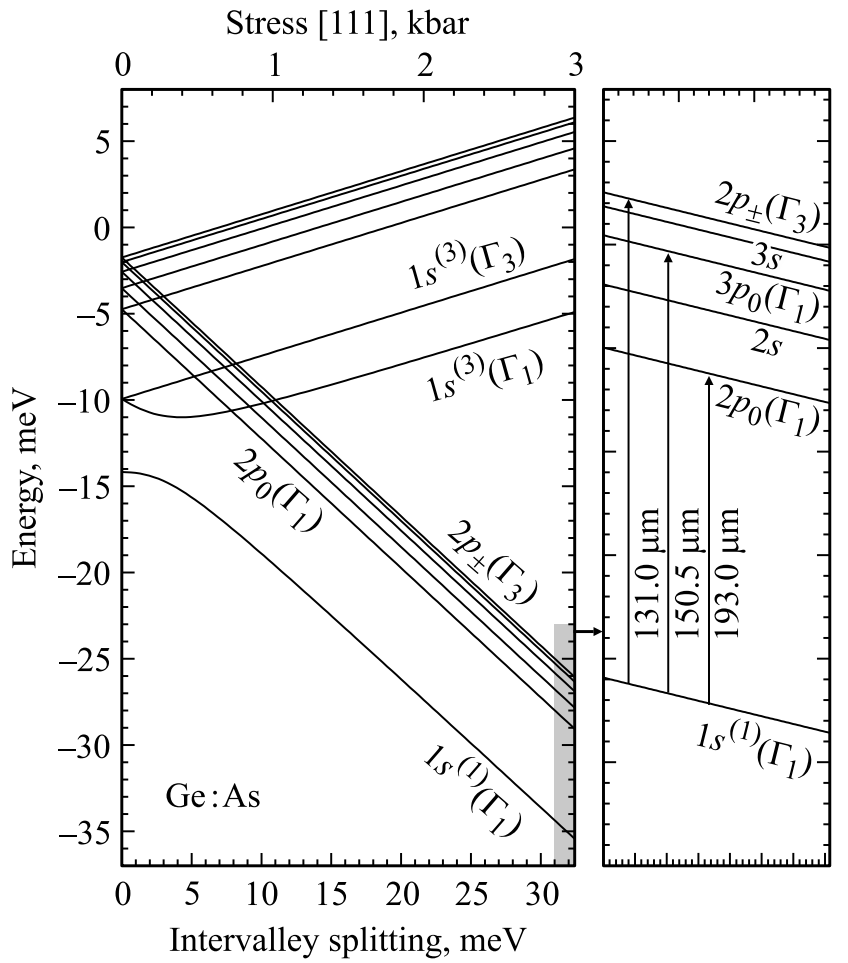

Рис. 1. Схема уровней донора мышьяка в германии при деформации $(\Delta>0)$ в кристаллографическом направлении [111]. Давление в 1 кбар соответствует междолинному расщеплению $\sim 10.81$ мэВ.

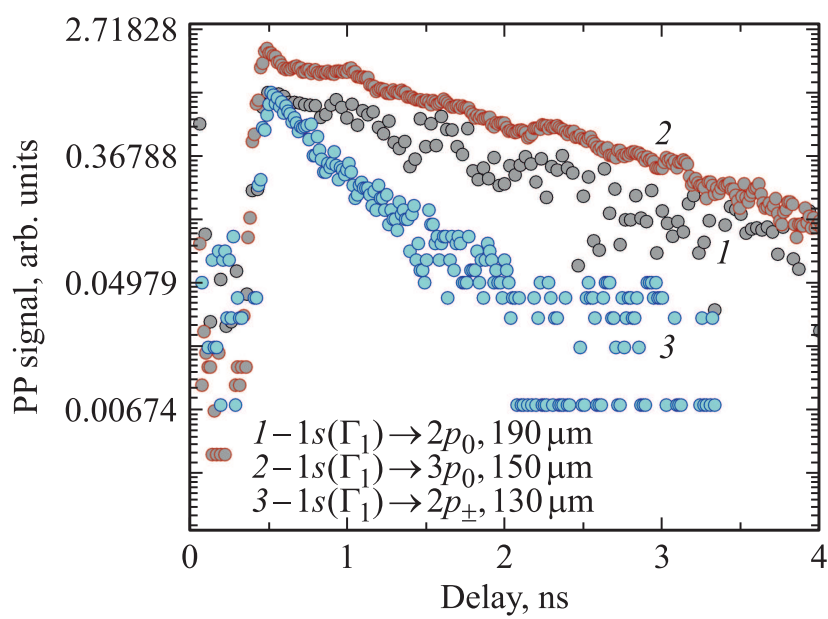

Рис. 2. Сигнал в системе накачка-зондирование при возбуждении различных уровней донора мышьяка в германии при $T=4$ К и $S=2.5$ кбар.

показывает экспоненциальный спад с характерным временем 1.3 нс (рис. 2). Здесь же на рис. 2 представлен результат измерения сигнала (pump-probe, PP signal) для $3 p_{0}$-состояния (энергия связи 8.6 мэВ, используемая длина волны накачки $1 s\left(\Gamma_{1}\right) \rightarrow 3 p_{0}$ при $S \approx 2.5$ кбар $\lambda=150$ мкм) при температуре $\sim 4 \mathrm{~K}$. Временна́я зависимость сигнала достаточно хорошо аппроксимируется экспоненциальной функцией с характерным вре- 


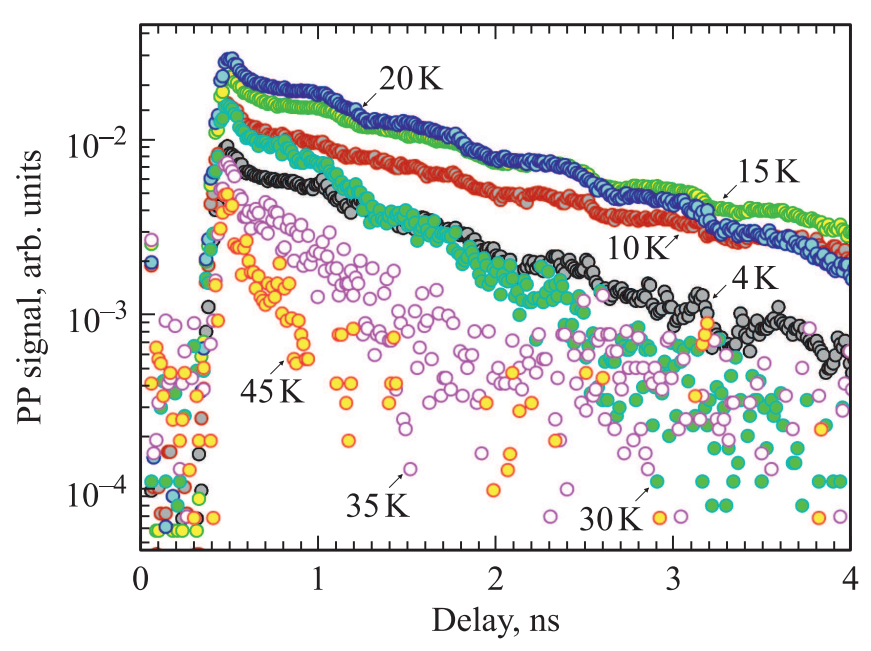

Рис. 3. Сигнал в системе накачка-зондирование при возбуждении состояния $3 p_{0}$ донора мышьяка в германии при различных температурах.

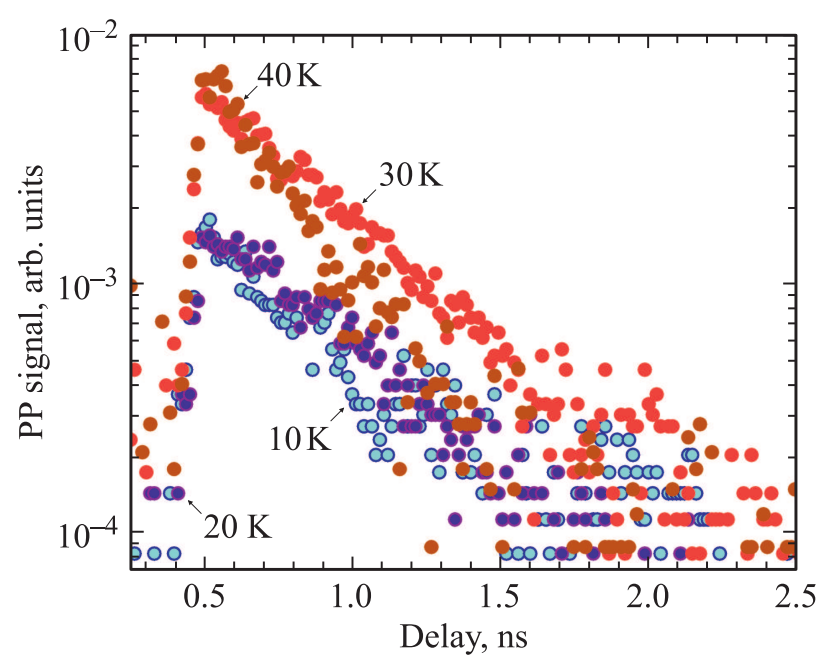

Рис. 4. Сигнал в системе накачка-зондирование при возбуждении состояния $2 p_{ \pm}$донора мышьяка в германии при различных температурах.

менем $\sim 1.3$ нс. На рис. 3 представлены зависимости исследуемого сигнала от температуры в диапазоне $T \approx 4-45 \mathrm{~K}$ для случая возбуждения $3 p_{0}$.

Случай фотовозбуждения $2 p_{ \pm}$-состояния представлен на рис. 2 (энергия связи 9.4 мэВ, $1 s\left(\Gamma_{1}\right) \rightarrow 2 p_{ \pm}$, при $S \approx 2.5$ кбар $\lambda=130$ мкм). Для сигнала характерен более быстрый экспоненциальный спад с характерным масштабом 0.4 нс. Влияние температуры на сигнал представлено на рис. 4.

\section{3. Обсуждение}

При одноосной деформации $S \approx 2.5$ кбар состояние $2 p_{0}$ является самым нижним среди возбужденных уровней донора, поэтому экспериментально измеренное вре- мя отклика $1.3 \mathrm{Hc}$ соответствует времени жизни $2 p_{0}$. Вычисление этого времени дает меньшее, но близкое значение, $\sim 1 \mathrm{Hc}[12]$. Сравнение с экспериментальным значением в отсутствие одноосного давления $(0.8$ нс) [9] показывает увеличение времени релаксации с деформацией, что качественно совпадает с результатом теоретических оценок. В недеформированном германии время релаксации уровня $2 p_{0}$ связано с переходами не только в основное состояние, $2 p_{0} \rightarrow 1 s^{(1)}\left(\Gamma_{1}\right)$, но и в состояние триплета, $2 p_{0} \rightarrow 1 s^{(3)}\left(\Gamma_{5}\right)$, энергия связи триплета 9-94 мэВ. Последнее при давлении в 2.5 кбар вдоль направления [111] оказывается выше по энергии, чем нижняя компонента уровня $2 p_{0}$, и не участвует в релаксации, увеличивая время жизни $2 p_{0}$. Некоторая компенсация возникает за счет уменьшения энергетического зазора между $2 p_{0}$ и основным уровнем донора, так как вероятность акустической релаксации растет с уменьшением энергии перехода.

Состояние $3 p_{0}$ располагается выше $2 p_{0}$ и $2 s$ (рис. 1 ). Поскольку измеренное время релаксации $3 p_{0}$ совпадает с $2 p_{0}$ (с точностью до временно́го разрешения метода $\sim 0.15 \mathrm{Hc})$, можно предположить, что релаксация идет через $2 p_{0}$, причем темп перехода $3 p_{0} \rightarrow 2 p_{0}$ должен быть бо́льшим в сравнении с величиной 1.3 нс. Таким образом, время распада состояния $3 p_{0}$ не превышает 0.15-0.2 нс (временно́е разрешение установки). Расчет темпов безызлучательной релаксации состояния $3 p_{0}$, выполненный в работе [11], также указывает на преимущественный канал релаксации $3 p_{0} \rightarrow 2 p_{0} \rightarrow 1 s\left(\Gamma_{1}\right) \mathrm{c}$ соответствующими временами $\sim 0.15$ и $\sim 1$ нс. Прямой же переход $3 p_{0} \rightarrow 1 s\left(\Gamma_{1}\right)$ в случае $S \approx 2.5$ кбар оказывается подавленным.

Другая ситуация наблюдается при накачке в состояние $2 p_{ \pm}$. Время релаксации, полученное в эксперименте, составляет $\sim 0.4 \mathrm{Hc}$, что близко к значению в недеформированном кристалле, где экспериментальная оценка дает не более 0.6 нс [9]. Поскольку $2 p_{ \pm}$лежит выше $3 p_{0}$ и $2 p_{0}$, измеренный темп релаксации должен определяться переходом в основное $s$-состояние, $2 p_{ \pm} \rightarrow 1 s\left(\Gamma_{1}\right)$, a не каналом релаксации, включающим $3 p_{0}$ и $2 p_{0}$. (Возможно, промежуточным в этом процессе может оказаться уровень $2 s$ или $3 s$, однако на дальнейших рассуждениях это не отразится.) Такое поведение отличается от случая $\mathrm{Sb}$, где экспериментально наблюдалось увеличение времени релаксации $2 p_{ \pm}$до 3.2 нс в одноосносдавленном германии, а основной канал релаксации был определен как $2 p_{ \pm} \rightarrow 3 p_{0} \rightarrow 2 p_{0} \rightarrow 1 s\left(\Gamma_{1}\right)$. Поскольку возбужденные состояния различных мелких доноров германия фактически идентичны, темпы релаксационных процессов между верхними возбужденными состояниями $2 p_{ \pm} \rightarrow 3 p_{0} \rightarrow 2 p_{0}$ в разных донорах также схожи. Различие этих примесей заключается в положении уровня $1 s\left(\Gamma_{1}\right)$, что также указывает на наличие прямого перехода в основное состояние в случае мышьяка.

Экспериментальное наблюдение высокого темпа релаксации для высоко лежащего состояния $2 p_{ \pm}$является неожиданным результатом. Так как при достаточ- 
но большой деформации вдоль кристаллографического направления [111] волновые функции как основного, так и возбуждаемого состояний доноров образованы вкладом лишь одной долины (вдоль которой приложено давление), междолинные процессы электрон-фононного взаимодействия не могут давать вклад в релаксацию состояний примесного центра. Таким образом, необходимо допустить, что релаксация определяется внутридолинными переходами с испусканием акустических фононов с большим волновым вектором (0.5-1 от размера зоны Бриллюэна).

Как известно, вероятность релаксации достаточно быстро спадает с увеличением волнового вектора фонона (следовательно, и энергии перехода), когда он начинает превосходить радиусы локализации волновых функций состояний доноров в обратном пространстве. Следовательно, при использовании волновых функций примеси, вычисленных в приближении эффективных масс с гамильтонианом, содержащим чисто кулоновский потенциал, вероятность релаксации должна быть мала. Таким образом, можно предположить, что в донорах мышьяка в германии велика роль короткодействующего потенциала (потенциала центральной ячейки), который обеспечивает медленное спадание волновой функции $\Psi(\mathbf{k})$ в обратном пространстве с увеличением волнового вектора $\mathbf{k}$ и обеспечивает возможность взаимодействия с фононами с большим волновым вектором. Предположение о роли короткодействующего потенциала частично основано на данных о самом большом среди доноров V группы долинно-орбитальном расщеплении ( 40\% от энергии триплета). Другой важной особенностью является то, что в области энергий, соответствующих переходу $2 p_{ \pm} \rightarrow 1 s\left(\Gamma_{1}\right)$, в сдавленном германии плотность состояний фононных поперечных акустических (ТА) мод сильно возрастает [13], что усиливает обоснованность сделанных предположений о существенности внутридолинных переходов с излучением фононов, волновой вектор которых сравним с размером зоны Бриллюэна кристалла.

В представленных температурных зависимостях (рис. 3) для уровня $3 p_{0}$ наблюдается увеличение темпа релаксации с ростом температуры, что вполне ожидаемо и связано с возросшей ролью индуцированных процессов. В случае уровня $2 p_{ \pm}$(рис. 4) такая зависимость не выглядит столь явной, по-видимому, частично вследствие того, что время релаксации при температурах $<10 \mathrm{~K}$ уже достаточно малое, составляет 3-4 длительности импульса возбуждения.

\section{4. Заключение}

В заключение можно отметить, что в условиях одноосного давления вдоль [111], когда волновые функции нижних состояний донора можно описывать как однодолинные, проведено измерение времен релаксации возбужденных состояний мышьяка в германии. Изме- рения выполнялись методом накачка-зондирование с использованием излучения лазера на свободных электронах. Накачка состояний осуществлялась из основного состояния $1 s\left(A_{1}\right)$. Показано, что время распада состояния $2 p_{0}$ составляет $1.3 \mathrm{Hc}, 3 p_{0}-$ не более $0.2 \mathrm{Hc}$, $2 p_{ \pm}-0.4$ нс. В случае релаксации уровня $2 p_{ \pm}$можно сделать вывод о наличии быстрого канала релаксации в основное состояние донора с участием внутридолинных ТА фононов.

\section{Благодарности}

Авторы благодарят за участие в работе операторов лазера на свободных электронах NovoFEL. Работа выполнена в ИЯФ СО РАН с использованием оборудования ЦКП „СЦСТИ“ на базе УНУ „Новосибирский ЛСЭ“.

\section{Финансирование работы}

Работа проведена при поддержке Российского научного фонда (проект № 19-72-20163).

\section{Конфликт интересов}

Авторы заявляют, что у них нет конфликта интересов.

\section{Список литературы}

[1] A.K. Ramdas, S. Rodriguez. Rep. Prog. Phys., 44, 1297 (1981).

[2] К.А. Ковалевский, Н.В. Абросимов, Р.Х. Жукавин, С.Г. Павлов, Г.-В. Хьюберс, В.В. Цыпленков, В.Н. Шастин. Квант. электрон., 45, 113 (2015).

[3] K.J. Morse, R.J.S. Abraham, A. DeAbreu, C. Bowness, T.S. Richards, H. Riemann, N.V. Abrosimov, P. Becker, H.-J. Pohl, M.L.W. Thewalt, S. Simmons. Sci. Adv., 3, e1700930 (2017).

[4] N. Deßmann, S.G. Pavlov, A. Pohl, N.V. Abrosimov, S. Winnerl, M. Mittendorff, R.Kh. Zhukavin, V.V. Tsyplenkov, D.V. Shengurov, V.N. Shastin, H.-W. Hübers. Appl. Phys. Lett., 106, 171109 (2015).

[5] Б.М. Гершензон, Г.Н. Гольцман, Н.Г. Птицина. Письма ЖЭТФ, 25, 574 (1977).

[6] F.A. Hegmann, J.B. Williams, B. Cole, M.S. Sherwin, J.W. Beeman, E.E. Haller. Appl. Phys. Lett., 76, 262 (2000).

[7] N. Deßmann, S.G. Pavlov, V.V. Tsyplenkov, E.E. Orlova, A. Pohl, V.N. Shastin, R.Kh. Zhukavin, S. Winnerl, M. Mittendorff, J.M. Klopf, N.V. Abrosimov, H. Schneider, H.-W. Hübers. Phys. Status Solidi B, 254, 1600803 (2017).

[8] Р.Х. Жукавин, К.А. Ковалевский, С.М. Сергеев, Ю.Ю. Чопорова, В.В. Герасимов, В.В. Цыпленков, Б.А. Князев, Н.В. Абросимов, С.Г. Павлов, В.Н. Шастин, Г. Шнайдер, Н. Дессманн, О.А. Шевченко, Н.А. Винокуров, Г.Н. Кулипанов, Г.-В. Хьюберс. Письма ЖЭТФ, 106, 555 (2017).

[9] Р.Х. Жукавин, К.А. Ковалевский, Ю.Ю. Чопорова, В.В. Цыпленков, В.В. Герасимов, П.А. Бушуйкин, Б.А. Князев, Н.В. Абросимов, С.Г. Павлов, Г.-В. Хьюберс, В.Н. Шастин. Письма ЖЭТФ, 110, 677 (2019).

[10] N. Deßmann, S.G. Pavlov, V.N. Shastin, R.Kh. Zhukavin, V.V. Tsyplenkov, S. Winnerl, M. Mittendorff, N.V. Abrosimov, H. Riemann, H.-W. Hübers. Phys. Rev. B, 89, 035205 (2014). 
[11] Yu.Yu. Choporova, V.V. Gerasimov, B.A. Knyazev, S.M. Sergeev, O.A. Shevchenko, R.Kh. Zhukavin, N.V. Abrosimov, K.A. Kovalevsky, V.K. Ovchar, H.-W. Hübers, G.N. Kulipanov, V.N. Shastin, H. Schneider, N.A. Vinokurov. Phys. Procedia, 84, 152 (2016).

[12] В.В. Цыпленков, В.Н. Шастин. ФТП, 52, 1469 (2018).

[13] P. Giannozzi, S. de Gironcoli, P. Pavone, S. Baroni. Phys. Rev. B, 43, 7231 (1991).

Редактор Л.В. Шаронова

\section{Relaxation of excited states of arsenic in stressed germanium}

K.A. Kovalevsky ${ }^{1}$, Yu.Yu. Choporova ${ }^{2,3}$, R.Kh. Zhukavin $^{1}$, N.V. Abrosimov' ${ }^{4}$, S.G. Pavlov ${ }^{5}$, H.-W. Hübers ${ }^{5,6}$, V.V. Tsyplenkov ${ }^{1}$, V.D. Kukotenko ${ }^{2,3}$, B.A. Knyazev ${ }^{2,3}$, V.N. Shastin ${ }^{1}$

${ }^{1}$ Institute for Physics of Microstructures, 603950 Nizhny Novgorod, Russia

${ }^{2}$ Budker Institute of Nuclear Physics,

Siberian Branch of Russian Academy of Sciences,

630090 Novosibirsk, Russia

${ }^{3}$ Novosibirsk State University, 630090 Novosibirsk, Russia

${ }^{4}$ Leibniz Institute of Crystal Growth, 12489 Berlin, Germany

${ }^{5}$ Institute of Optical Sensor Systems, German Aerospace Center (DLR), 12489 Berlin, Germany

${ }^{6}$ Institut für Physik, Humboldt-Universität zu Berlin, 12489 Berlin, Germany

Abstract The relaxation times of the lower $p$ states of the arsenic donor in a germanium crystal deformed along the [111] crystallographic direction are studied. The measurements were performed by the „pump-probe“ method using $\mathrm{THz}$ radiation of a free-electron laser. The states were pumped from the ground state $1 s\left(A_{1}\right)$. The measured decay time of the $2 p_{0}$ state is $1.3 \mathrm{~ns}$, $3 p_{0}-$ not more than $0.2 \mathrm{~ns}, 2 p_{ \pm}-0.4 \mathrm{~ns}$. It was shown that a relatively high rate of relaxation of the $2 p_{ \pm}$state is associated with interactions with intra-valley TA phonons. 